\title{
Correlation between C-reactive Protein with Malondialdehyde in Systemic Lupus Erythematosus Patients
}

\author{
Nur Atik $\mathbb{D}^{1,2}$ S. Putri Pratiwi, ${ }^{3}$ and Laniyati Hamijoyo ${ }^{2,4}$ \\ ${ }^{1}$ Department of Biomedical Sciences, Faculty of Medicine, Padjadjaran University, Bandung, Indonesia \\ ${ }^{2}$ Lupus Study Centre, Immunology Study Group, Padjadjaran University/Hasan Sadikin Hospital, Bandung, West Java, Indonesia \\ ${ }^{3}$ Undergraduate Program, Faculty of Medicine, Padjadjaran University, Bandung, Indonesia \\ ${ }^{4}$ Division of Rheumatology, Department of Internal Medicine, Faculty of Medicine, \\ Padjadjaran University/Dr. Hasan Sadikin General Hospital Bandung, Indonesia \\ Correspondence should be addressed to Nur Atik; n.atik@unpad.ac.id
}

Received 26 March 2020; Revised 7 June 2020; Accepted 9 June 2020; Published 1 July 2020

Academic Editor: Charles J. Malemud

Copyright (C) 2020 Nur Atik et al. This is an open access article distributed under the Creative Commons Attribution License, which permits unrestricted use, distribution, and reproduction in any medium, provided the original work is properly cited.

\begin{abstract}
Systemic lupus erythematosus (SLE) is a systemic autoimmune disease characterized by an inflammatory process. One of the inflammation markers that can be measured is C-reactive protein (CRP). Another indicator of inflammation is malondialdehyde (MDA), though it is still uncommon to be analyzed in SLE patients. The study looked for the MDA value and found a correlation with CRP. A cross-sectional study design with a correlative analytical was performed. CRP level data was taken from Hasan Sadikin lupus registry data, and MDA levels were analyzed from a bioarchive patient's serum. We collected the patients' data who had CRP level from Hasan Sadikin lupus registry and analysed MDA levels from the serum sample. MDA levels were analyzed using an ELISA method. The data obtained were analyzed using the Pearson correlation and Eta correlation test. The study involved 78 data patients as subjects. It was found that the median of CRP and MDA was $0.85 \mathrm{mg} / \mathrm{l}$ and $153.10 \mathrm{ng} / \mathrm{ml}$, respectively. These results indicate that the CRP levels in SLE patients are still within normal limits. Statistical analysis showed no correlation between CRP and MDA level $(r=0.2, P>0.05)$. Additionally, the correlation between CRP and MDA with organ involvement, such as lupus nephritis (LN), lupus cutaneous (LC), and lupus musculoskeletal (LM), showed no correlation $\left(F_{\mathrm{h}}<F_{\mathrm{t}}\right)$. There is no correlation between CRP and MDA levels in SLE patients, and specific organ involvement of the disease does not affect the correlation.
\end{abstract}

\section{Introduction}

Systemic lupus erythematosus (SLE) is a systemic, chronic, and multisystem autoimmune disease that attacks various organs of the body caused by the deposition of immune complexes $[1,2]$. This immune complex is caused by the failure of self-tolerance of B and $\mathrm{T}$ lymphocytes as well as the number of nuclear antigens originating from the fault in clearing dead cells which resulted from apoptosis, which causes a systemic inflammatory response and manifests according to the affected tissue or organ [1,3-5]. Various inflammatory markers can be measured in SLE, one of which is C-reactive protein (CRP). A study states that CRP levels could present as normal or elevated in SLE patients as a sign of an inflammatory response [6]. CRP has been commonly used as a marker for inflammation, infection, tissue damage, malignancy, and autoimmune diseases [7].

CRP is a plasma protein synthesized by the liver, and their numbers increase during infections and inflammation. The presence of phagocytic cells that release IL- 6 and IL-1 induces CRP production as a result of immune response [8]. Another indicator of oxidative stress and inflammation in SLE patients is malondialdehyde (MDA) [4]. Previous studies have shown a significant increase in MDA levels in SLE patients $[4,9]$. MDA is a reactive aldehyde as the final result of a fat peroxidation process due to elevated levels of reactive oxygen species (ROS) $[3,4,9]$. ROS is generally formed as a result of cell metabolism in the body in small amounts. However, it causes harmful effects in excessive amount [10]. Other studies found that excess amount or 
production of ROS in SLE patients can be caused by several factors, such as an inflammatory response $[3,4,9,10]$. These compounds then react with lipid membrane of the cells which become one of the targets prone to be damaged by ROS and produce hazardous compounds including MDA $[4,10]$.

The correlation between inflammatory conditions with the oxidation process is still poorly understood, specifically a relationship between the level of inflammation characterized by CRP levels and oxidative stress characterized by MDA levels in SLE patients. Previous studies have found that increased ROS correlates with CRP in lupus nephritis patients, and there is a correlation between inflammatory conditions that trigger oxidation in SLE patients [3]. Thus, we analyzed the CRP and MDA levels from the SLE patients to know the correlation for both inflammatory markers. This research is expected to be a source of data on CRP and MDA levels, especially MDA that are not included in the routine examination in SLE patients. It is also expected to find information about the correlation between CRP and MDA in SLE patients.

\section{Materials and Methods}

2.1. The Patients' Data Collection. The cross-sectional analytic study using clinical, CRP data and serum bioarchived samples from Hasan Sadikin Lupus Registry research, whereas CRP levels were taken from Hasan Sadikin Lupus Registry data [11]. We collected 78 patients' data that have been included in the inclusion criteria. The patients' data were registered in the Rheumatology Division of the Department of Internal Medicine Hasan Sadikin Hospital Bandung from January 1, 2014, to December 31, 2018, period. The exclusion criteria consist of patients with incomplete lupus data registry, and the serum samples were damaged. The minimum sample size needed for this research is 62 , which is determined by the sample size formula for correlative analytics research.

This study is following the WMA Declaration of Helsinki on ethical principles for medical research, and the protocol was approved by the Research Ethics Committee of Padjadjaran University (685/UN6.KEP/EC/2019).

2.2. ELISA Procedure. An ELISA competitive method was used for MDA level testing in this study, conducted at the Clinical Pathology Laboratory of Hasan Sadikin Hospital Bandung. 78 serum samples from the subject bioarchive were proceeded to MDA analysis. All the sera were matched with the CRP value prior the MDA analysis. The serum samples had previously been frozen at $-80^{\circ} \mathrm{C}$ and then thawed by placing the tube to room temperature $\left(18-25^{\circ} \mathrm{C}\right)$ for 2 hours.

MDA was measured using an ELISA Kit Elabscience (according to the manufacturer's instructions) [12]. Briefly, $50 \mu \mathrm{L}$ of serum were tested in each well by adding $50 \mu \mathrm{L}$ of biotinylated detection antibody. It was followed by incubation for 45 minutes at $37^{\circ} \mathrm{C}$. The reaction was washed three times. Add $100 \mu \mathrm{L}$ of HRP conjugate, incubate for 30 minutes at $37^{\circ} \mathrm{C}$, and then wash five times. Add $90 \mu \mathrm{L}$ of substrate reagent then incubate for 15 minutes at $37^{\circ} \mathrm{C}$. Add $50 \mu \mathrm{L}$ stop
TABLE 1: SLE patient characteristics.

\begin{tabular}{|c|c|}
\hline Variable & $\begin{array}{l}\text { SLE patients } \\
\quad(n=78)\end{array}$ \\
\hline \multicolumn{2}{|l|}{ Gender: } \\
\hline (1) Male & $1(1.28 \%)$ \\
\hline (2) Female & $77(98.72 \%)$ \\
\hline Age $^{\mathrm{b}}$ & $39(9.55)$ \\
\hline $\begin{array}{l}\text { Duration or length of disease }{ }^{c} \text { (counted from the } \\
\text { date of diagnosis to the date the blood was drawn) }\end{array}$ & $4.5(1-18)$ \\
\hline \multicolumn{2}{|l|}{ Jobs $^{\mathrm{a}}$} \\
\hline (i) Housewives & $46(58.97 \%)$ \\
\hline (ii) Employees & $8(10.26 \%)$ \\
\hline (iii) Teachers & $7(8.97 \%)$ \\
\hline (iv) Private employees & $5(6.41 \%)$ \\
\hline (v) Students & $2(2.56 \%)$ \\
\hline (vi) Government employees & $1(1.28 \%)$ \\
\hline (vii) Professional & $1(1.28 \%)$ \\
\hline (viii) Others & $2(2.56 \%)$ \\
\hline (ix) Unemployed & $1(1.28 \%)$ \\
\hline (x) No data & $5(6.41 \%)$ \\
\hline \multicolumn{2}{|l|}{ Organ involvement $\mathrm{t}^{\mathrm{a}}$} \\
\hline (i) Lupus nephritis & $43(55.13 \%)$ \\
\hline (ii) Lupus cutaneous & $63(80.77 \%)$ \\
\hline (iii) Lupus musculoskeletal & $62(79.49 \%)$ \\
\hline \multicolumn{2}{|l|}{ SLEDAI-2 $\mathrm{k}^{\mathrm{a}}$} \\
\hline (i) $<4$ & $47(60.26 \%)$ \\
\hline (ii) $\geq 4$ & $31(39.74 \%)$ \\
\hline
\end{tabular}

${ }^{\mathrm{a} A m o u n t}$ (percentage). ${ }^{\mathrm{b}}$ Mean (average). ${ }^{\mathrm{c}}$ Median (minimum-maximum).

solution and determine the optical density (OD) value at $450 \mathrm{~nm}$; then calculate the results. The MDA detection limit with the Competitive ELISA Kit method is 31.25-2000 ng/mL.

2.3. Statistical Analysis. The data collected were analysed using IBM SPSS Statistics version 23.0 statistical software. The analysis of data correlation between CRP and MDA was done using the Pearson correlation coefficient. The correlation between CRP and MDA levels with organ involvement was analysed using the Eta correlation coefficient test.

\section{Results}

3.1. SLE Patient Characteristics. There were 78 CRP patient data obtained, which were connected with patients' serum who had met the criteria. Data characteristics of the subjects of this study showed as many as 77 (98.72\%) patients in this study were female. The average age of SLE patients in this study was 39 years. The duration or length of time the subject had is a median of 4.5 years. Most of the subjects work as a housewife (58.97\%). There were 43 (55.13\%) patients with kidney disorders involved in this study, 63 patients with skin disorders $(80.77 \%)$, and 62 patients with disorders of muscles and bones (79.49\%). The disease activity of SLE patients measured by SLEDAI-2 $\mathrm{k}$ showed that 47 patients $(60.26 \%)$ were in an inactive immune system (Table 1). 
3.2. Correlation between CRP and MDA. CRP and MDA levels of SLE patients in this study were found to have a median of $0.85 \mathrm{mg} / \mathrm{L}$ and $153.10 \mathrm{ng} / \mathrm{mL}$, respectively. The median of CRP results showed no increase of CRP levels in the subjects. The lowest-highest value of each variable was $0.05-27.3 \mathrm{mg} / \mathrm{L}$ and $50.88-693.16 \mathrm{ng} / \mathrm{mL}$. The analysis test showed the $r$ value of the correlation coefficient between the two variables was 0.2 with a $P$ value $>0.05$, so there was no correlation between CRP and MDA in SLE patients analyzed by the Pearson correlation method (Table 2).

3.3. Correlation between $C R P$ and MDA with Organ Involvement. A study in Brazil found that increased ROS correlated with CRP in lupus nephritis patients [3]. Increased free radicals were also found in proliferative lupus nephritis patients compared with lupus patients who did not have kidney abnormalities or lupus patients without nephritis. In lupus cutaneous patients, an increase in free radicals is in accordance with the activity of SLE disease, especially after exposure to ultraviolet light [13]. The occurrence of an imbalance of ROS with antioxidants in the body is what causes oxidative stress [14]. The excess production of ROS in the body is also considered to have a relationship with inflammatory conditions $[15,16]$. Therefore, to find out whether each organ involvement influences the concentration of CRP or MDA levels, the Eta correlation coefficient test was performed (Table 3).

Based on the results of the analysis, the coefficient of $\eta=0.127$ was obtained in the correlation of CRP with lupus nephritis, $\eta=0.083$ with lupus cutaneous, and $\eta=0.066$ with lupus musculoskeletal. Furthermore, the coefficient value $\eta=0.026$ was obtained in the correlation of MDA with lupus nephritis, $\eta=0.012$ with lupus cutaneous, and $\eta=0.114$ with lupus musculoskeletal. It means that CRP and MDA values with organ involvement in lupus patients in this study had a very low or weak correlation. Furthermore, we proceed the data with the $F$ test to know the correlation between CRP and MDA to the specific organs that affected in the SLE patients. We found that all results showed $F_{\text {count }}<F_{\text {table. }}$. It means that there is no significant correlation between CRP and MDA values with organ involvement in lupus patients (Table 3).

\section{Discussion}

This study shows that the prevalence of female SLE patients $(98.72 \%)$ is greater than that of men $(1.28 \%)$. These results are consistent with a previous study, which states that SLE attacks more women $[11,17]$. The average age of the subjects is 39 years. This is also in accordance with the same previous study that the highest numbers of SLE disease occurred in the age range of 30-49 years and 50-64 years [17]. The duration of lupus illness has a median of 4.5 years with a duration range of 1-18 years. These results are different from other studies which shows a range of duration of SLE disease ranging from 0 to 15 years with a median of 9 years [3]. Previous studies in West Java, Indonesia, had an average duration of illness of 76.5 months [11]. The involvement of organs in this study mostly attacks the skins $(80.77 \%)$ then
TABLE 2: Correlation coefficient of CRP and MDA.

\begin{tabular}{lcc}
\hline Variables & SLE patients $(n=78)$ & $r(P)$ \\
\hline $\mathrm{CRP}^{\mathrm{a}}$ & $0.85(0.05-27.30)$ & $0.2(0.08)$ \\
$\mathrm{MDA}^{\mathrm{a}}$ & $153.10(50.88-693.16)$ & \\
\hline
\end{tabular}

TABLE 3: Coefficient correlation of CRP or MDA with organ involvement.

\begin{tabular}{lcccc}
\hline Variable & $\eta$ & $F_{\text {count }}$ & $F_{\text {table }}$ & Interpretation \\
\hline CRP and LN correlation & 0.127 & 1.246 & 3.97 & $F_{\mathrm{h}}<F_{\mathrm{t}}$ \\
CRP and LC correlation & 0.083 & 0.527 & 3.97 & $F_{\mathrm{h}}<F_{\mathrm{t}}$ \\
CRP and LM correlation & 0.066 & 0.333 & 3.97 & $F_{\mathrm{h}}<F_{\mathrm{t}}$ \\
MDA and LN correlation & 0.026 & 0.051 & 3.97 & $F_{\mathrm{h}}<F_{\mathrm{t}}$ \\
MDA and LC correlation & 0.012 & 0.011 & 3.97 & $F_{\mathrm{h}}<F_{\mathrm{t}}$ \\
MDA and LM correlation & 0.114 & 1.001 & 3.97 & $F_{\mathrm{h}}<F_{\mathrm{t}}$ \\
\hline
\end{tabular}

LN: lupus nephritis; LC: lupus cutaneous; LM: lupus musculoskeletal.

followed by musculoskeletal disorders (79.49\%) and the kidneys $(55.13 \%)$. This study is slightly different from other studies which state that $85 \%$ of the complications are involved in musculoskeletal and arthritis, $75 \%$ in the skin and mucosa, and 30\% in kidney disorders [18].

A characteristic of SLE is the presence of an immune complex that activates the endosomal toll-like receptor (TLR) and triggers the inflammatory process. Various markers of inflammation can be measured in SLE patients, such as CRP [6]. CRP levels can be used as an indicator of low-grade inflammation in infections and chronic diseases [19-21]. The median value of CRP in this study is $0.85 \mathrm{mg} / \mathrm{L}$, known as the normal level [20]. A CRP level can be normal or elevated in SLE patients as a sign of an inflammatory response [6]. Other studies have shown that an average CRP level $>10 \mathrm{mg} / \mathrm{L}$ in both SLE patients who were active, inactive, exacerbation, infected, or not [22]. High CRP levels $>10 \mathrm{mg} / \mathrm{L}$ can indicate active or flare SLE patients $[23,24]$. A previous study showed an average CRP value of $323.5 \mathrm{mg} / \mathrm{dL}$ with a median of $48 \mathrm{mg} / \mathrm{dL}$, and others showed $3.41 \mathrm{mg} / \mathrm{L}$ and $1.57 \mathrm{mg} / \mathrm{L}$ [25-27]. Factors that can affect the diversity of high or low CRP levels include psychosocial stress, low education, antimalaria drugs, infections such as serositis, Body Mass Index (BMI), and ethnicity. It is known that the disease activity is correlated with the inflammatory response $[28,29]$. We showed that the majority of patients' serum samples were taken in an inactive stage of the immune system. It caused the CRP levels of patients in this study to be within normal limits.

In addition, most of the subjects in this study work as housewives. Based on the WHO's "Global Strategy on Diet, Physical Activity, and Health," housewife activities are categorized as moderate physical activity. Moderate-intensity physical activity can also reduce the inflammatory response by suppressing expressions of TLR 4 on monocytes [27]. The mechanism of physical activity affecting TLR expression is yet to be clearly known, but there is a possible role of antiinflammatory cytokines and heat shock protein (HSP) 
involvement [30,31]. Binding of HSP with TLR 4 can cause an inflammatory response. A study proves that physical activity can cause downregulation of HSP [32]. Another important factor influencing the inflammatory response is psychosocial stress. It causes the secretion of proinflammatory cytokines (IL-1 and IL-6) and stress hormones (corticosteroids and catecholamines) thereby triggering inflammatory processes and CRP [33]. The involvement of gene polymorphisms in CRP also affects the course of the disease and causes low CRP levels during flare conditions in SLE patients [23].

In SLE patients, an increase of ROS affects the severity of the disease $[3,34,35]$. The ROS causes oxidative stress conditions, reactive autoantibodies, autoantigen production, and immune cell disorders $[3,13]$. The imbalance of ROS levels with body antioxidants causes oxidative stress conditions and triggers fat peroxidation reactions and produces the MDA $[3,4,10,16]$. In this study, the median of MDA value in patients was $153.10 \mathrm{ng} / \mathrm{mL}$. Other research in Iran shows a median MDA level of $1.84 \mu \mathrm{mol} / \mathrm{L}$ and states that an increasing MDA level as an indicator of inflammation and oxidative stress is related to the presence of lupus disease [4]. The MDA levels may increase due to mitochondrial dysfunction in SLE patients, external exposure such as cigarettes and ultraviolet (UV) rays, and low levels of glutathione as an antioxidant in lupus patients. Previous studies found that there is a correlation between inflammatory conditions that trigger the oxidation process in SLE patients [3]. However, our results showed that there was no correlation between CRP and MDA in SLE patients in this study $(r=0.2, P>0.05)$.

Additionally, we tested the correlation between CRP and MDA levels in patients with lupus nephritis, lupus musculoskeletal, and lupus cutaneous. Previous studies suggest that increased ROS correlates with CRP in lupus nephritis patients [3]. Other studies state that oxidative stress triggers inflammatory processes and the incidence of lupus nephritis [36]. Increased free radicals are found in proliferative lupus nephritis patients compared with lupus patients without kidney disorders. Besides, an increase in free radicals also occurs in the skin organs of lupus cutaneous patients, which happens in accordance with SLE, especially after exposure to ultraviolet rays [13]. However, among the three analysis results, we did not find any correlation between both CRP and MDA levels with organ involvement $\left(F_{\text {count }}<F_{\text {table }}\right)$. CRP as a marker of inflammation can still be found within normal limits in SLE patients. As an additional, there is no correlation between CRP and MDA in SLE patients, and also, there is no correlation between CRP and MDA levels with organ involvement.

This first study regarding CRP and MDA correlation in Indonesia SLE patients has several limitations. The recent study only focuses on the status of CRP and MDA without observing deeply for the infection status and the treatment history as well. The limited number of subjects could also be a limitation for this study.

\section{Conclusion}

CRP as a marker of inflammation can still be found within normal limits in SLE patients. As an additional, there is no correlation between CRP and MDA in SLE patients, and also, there is no correlation between CRP and MDA levels with organ involvement.

\section{Abbreviations}

SLE: $\quad$ Systemic lupus erythematosus

CRP: C-reactive protein

MDA: Malondialdehyde

ELISA: Enzyme-Linked Immunosorbent Assay

ROS: $\quad$ Reactive oxygen species

HRP: Horseradish peroxidase

OD: Optical density

SLEDAI: Systemic Lupus Erythematosus Disease Activity Index

LN: Lupus nephritis

LC: $\quad$ Lupus cutaneous

LM: Lupus musculoskeletal

TLR: Toll-like receptor

HSP: Heat shock protein.

\section{Data Availability}

The data used to support the findings of this study are available from the corresponding author upon request.

\section{Conflicts of Interest}

The authors declare that there is no conflicts of interest regarding publication of this paper.

\section{Acknowledgments}

The author (NA) thank the DIKTI grant. Researchers would like to thank the SLE Study Group of Rheumatology Division of the Department of Internal Medicine and the Clinical Pathology Laboratory Team at Hasan Sadikin General Hospital Bandung for their assistance in completing the research data.

\section{References}

[1] A. K. Abbas, A. H. Lichtman, and S. Pillai, Cellular and Molecular Immunology, Elsevier Saunders, Canada, 8th edition, 2015.

[2] V. Kumar, A. K. Abbas, and J. C. Aster, Robbins and Cotran Pathologic Basis of Disease, Elsevier Saunders, Philadelphia, 9th edition, 2015.

[3] M. Lozovoy, A. Simão, C. Panis et al., "Oxidative stress is associated with liver damage, inflammatory status, and corticosteroid therapy in patients with systemic lupus erythematosus," Lupus, vol. 20, no. 12, pp. 1250-1259, 2011.

[4] M. Tanhapour, A. Vaisi-Raygani, F. Bahrehmand et al., "Association between the cytotoxic T-lymphocyte antigen-4 mutations and the susceptibility to systemic lupus erythematosus; contribution markers of inflammation and oxidative stress," Cellular and Molecular Biology, vol. 62, no. 12, pp. 56-61, 2016.

[5] S. Pathak and C. Mohan, "Cellular and molecular pathogenesis of systemic lupus erythematosus: lessons from animal models," Arthritis Research \& Therapy, vol. 13, no. 5, pp. 241-249, 2011. 
[6] B. H. Rovin, C. C. Mok, D. J. Birmingham, L. A. Hebert, and L. Y. Ho, "High-sensitivity C-reactive protein, disease activity, and cardiovascular risk factors in systemic lupus erythematosus," Arthritis Care \& Research, vol. 65, no. 3, pp. 441-447, 2013.

[7] W. M. Fakanya and I. E. Tothill, "Detection of the inflammation biomarker C-reactive protein in serum samples: towards an optimal biosensor formula," Biosensors, vol. 4, no. 4, pp. 340-357, 2014.

[8] S. Kany, J. T. Vollrath, and B. Relja, "Cytokines in inflammatory disease," International Journal of Molecular Sciences, vol. 20, no. 23, p. 6008, 2019.

[9] U. Hardt, A. Larsson, I. Gunnarsson et al., "Autoimmune reactivity to malondialdehyde adducts in systemic lupus erythematosus is associated with disease activity and nephritis," Arthritis Research \& Therapy, vol. 20, no. 36, pp. 1-12, 2018.

[10] D. Shah, N. Mahajan, S. Sah, S. K. Nath, and B. Paudyal, "Oxidative stress and its biomarkers in systemic lupus erythematosus," Journal of Biomedical Science, vol. 21, no. 1, pp. 1-13, 2014.

[11] L. Hamijoyo, S. Candrianita, A. R. Rahmadi et al., "The clinical characteristics of systemic lupus erythematosus patients in Indonesia: a cohort registry from an Indonesiabased tertiary referral hospital," Lupus, vol. 28, no. 13, pp. 1604-1609, 2019.

[12] N. A. A. Nasir, R. Agarwal, S. H. S. A. Kadir et al., "Reduction of oxidative-nitrosative stress underlies anticataract effect of topically applied tocotrienol in streptozotocin-induced diabetic rats," PLoS One, vol. 12, no. 3, article e0174542, 2017.

[13] J. C. Oates, "The biology of reactive intermediates in systemic lupus erythematosus," Autoimmunity, vol. 43, no. 1, pp. 5663, 2009

[14] T. L. De Jager, A. E. Cockrell, and S. S. Du Plessis, "Ultraviolet light induced generation of reactive oxygen species," in Ultraviolet Light in Human Health, Diseases and Environment, pp. 15-23, Springer, Cham, 2017.

[15] A. El-Kenawi and B. Ruffell, "Inflammation, ROS, and mutagenesis," Cancer Cell, vol. 32, no. 6, pp. 727-729, 2017.

[16] T. A. Gheita and S. A. Kenawy, "Measurement of malondialdehyde, glutathione, and glutathione peroxidase in SLE patients," in Systemic Lupus Erythematosus, Humana Press, New York, 2014.

[17] C. H. Feldman, L. T. Hiraki, J. Liu et al., "Epidemiology and sociodemographics of systemic lupus erythematosus and lupus nephritis among US adults with Medicaid coverage, 20002004," Arthritis and Rheumatism, vol. 65, no. 3, pp. 753-763, 2013.

[18] L. Lisnevskaia, G. Murphy, and D. Isenberg, "Systemic lupus erythematosus," Lancet, vol. 384, no. 9957, pp. 1878-1888, 2014.

[19] D. González-Fernández, E. C. Pons, D. Rueda et al., "C-reactive protein is differentially modulated by co-existing infections, vitamin deficiencies and maternal factors in pregnant and lactating indigenous Panamanian women," Infectious Diseases of Poverty, vol. 6, no. 94, pp. 1-14, 2017.

[20] A. S. Fauci and C. A. Langford, Harrison's Rheumatology, McGraw-Hill, New York, USA, 3rd edition, 2013.

[21] T. A. Gottschalk, E. Tsantikos, and M. L. Hibbs, "Pathogenic inflammation and its therapeutic targeting in systemic lupus erythematosus," Frontiers in immunology, vol. 6, 2015.
[22] J. Batuca and J. Delgado Alves, "C-reactive protein in systemic lupus erythematosus," Autoimmunity, vol. 42, no. 4, pp. 282$285,2009$.

[23] A. Dima, D. Opris, C. Jurcut, and C. Baicus, "Is there still a place for erythrocyte sedimentation rate and C-reactive protein in systemic lupus erythematosus?," Lupus, vol. 25, no. 11, pp. 1173-1179, 2016.

[24] Q. Zhang, D. Q. Ye, G. P. Chen, and Y. Zheng, "Oxidative protein damage and antioxidant status in systemic lupus erythematosus," Clinical and Experimental Dermatology, vol. 35, no. 3, pp. 287-294, 2010.

[25] Z. Albar and L. K. Wijaya, "Is there a relationship between serum C-reactive protein level and dyslipidaemia in systemic lupus erythematosus?," Acta Medica Indonesiana, vol. 38, no. 1, pp. 23-28, 2006.

[26] P. Hu, E. H. Herningtyas, V. Kale et al., "External quality control for dried blood spot-based c-reactive protein assay: experience from the Indonesia family life survey and the longitudinal aging study in India," Biodemography and Social Biology, vol. 61, no. 1, pp. 111-120, 2015.

[27] A. Maharani, "Socio-economic inequalities in C-reactive protein levels: evidence from longitudinal studies in England and Indonesia," Brain, Behavior, and Immunity, vol. 82, pp. 122-128, 2019.

[28] A. M. Eudy, A. I. Vines, M. A. Dooley, G. S. Cooper, and C. G. Parks, "Elevated C-reactive protein and self-reported disease activity in systemic lupus erythematosus," Lupus, vol. 23, no. 14, pp. 1460-1467, 2014.

[29] L. Pan, M. P. Lu, J. H. Wang, M. Xu, and S. R. Yang, "Immunological pathogenesis and treatment of systemic lupus erythematosus," World Journal of Pediatrics, vol. 16, no. 1, pp. 19-30, 2020.

[30] X. D. Wu, K. Zeng, W. L. Liu et al., "Effect of aerobic exercise on miRNA-TLR4 signaling in atherosclerosis," International Journal of Sports Medicine, vol. 35, no. 4, pp. 344-350, 2014.

[31] M. Gleeson, B. McFarlin, and M. Flynn, "Exercise and toll-like receptors," Exerisec Immunology Review, vol. 12, pp. 34-53, 2006.

[32] D. Simar, D. Malatesta, S. Badiou, A. M. Dupuy, and C. Caillaud, "Physical activity modulates heat shock protein72 expression and limits oxidative damage accumulation in a healthy elderly population aged 60-90 years," The Journals of Gerontology Series A: Biological Sciences and Medical Sciences, vol. 62, no. 12, pp. 1413-1419, 2007.

[33] P. H. Black and L. D. Garbutt, "Stress, inflammation and cardiovascular disease," Journal of Psychosomatic Research, vol. 52, no. 1, pp. 1-23, 2002.

[34] Y. L. Lightfoot, L. P. Blanco, and M. J. Kaplan, "Metabolic abnormalities and oxidative stress in lupus," Current Opinion in Rheumatology, vol. 29, no. 5, pp. 442-449, 2017.

[35] L. Diebold and N. S. Chandel, "Mitochondrial ROS regulation of proliferating cells," Free Radical Biology and Medicine, vol. 100, pp. 86-93, 2016.

[36] A. Perl, "Oxidative stress in the pathology and treatment of systemic lupus erythematosus," Nature Reviews Rheumatology, vol. 9, no. 11, pp. 674-686, 2013. 